\title{
Can we infer the Initial Mass Function of galaxies at $\mathrm{z} \sim 2$ ?
}

\author{
Themiya Nanayakkara ${ }^{1,2}$, Karl Glazebrook ${ }^{1}$ and The ZFIRE Team ${ }^{3}$ \\ ${ }^{1}$ Centre for Astrophysics and Supercomputing, Swinburne University of Technology, \\ Hawthorn, Victoria 3122, Australia \\ ${ }^{2}$ email: tnanayak@astro.swin.edu.au \\ ${ }^{3}$ website: http://zfire.swinburne.edu.au
}

\begin{abstract}
Recent studies show increasing evidence for the possibility of the Initial Mass Function (IMF) of galaxies to vary as a function of numerous physical parameters. These studies mainly focus on the low redshift galaxies $($ at $z \leqslant 0.1)$ and is not backed by any studies at higher redshifts. Using MOSFIRE data from KECK, we use the $\mathrm{H} \alpha$ Equivalent-Width (EW) as a proxy for the IMF to study in situ IMF of galaxies at $z \sim 2$. In this proceedings we focus on the underlying physics of this method and present our initial findings. More work is needed to interpret the results accurately and will be presented in our future papers.
\end{abstract}

Keywords. dust, extinction, galaxies: clusters: general, galaxies: fundamental parameters, galaxies: high-redshift, galaxies: starburst, infrared: galaxies

\section{Introduction}

The IMF can be defined as the number of stars formed at the same time in some volume of space as a function of stellar mass in a single stellar population (Scalo (1986)). We expect the galaxy evolution to be mainly driven by the mass of the stars it incorporates. Therefore, the IMF plays a vital role in determining the evolution of galaxies and is fundamental in deriving many properties of galaxies such as the mass-to-light ratios etc. IMF is a purely empirical formula (Baldry (2003), Chabrier (2003), Kroupa (2001), Scalo (1986), Kennicutt (1983), Miller (1979), Salpeter (1955)) and historic studies found no statistically significant differences in the IMF from the Salpeter (1955) value.

Recent studies has started showing increasing evidence for a non-universal IMF (Ferreras (2013), Conroy (2013), Cappellari (2012), Meurer (2011), Gunawardhana (2011), vanDokkum (2010), Hoversten (2008)). All of these studies focus on galaxies at $\mathrm{z} \leqslant 0.1$.

To fully understand the properties of high redshift galaxies and how they evolve to present time, it is imperative to study the IMF of galaxies at higher redshifts. Some theoretical studies also suggest the possibility of the IMF to vary as a function of cosmic time (Ferreras (2015), Chattopadhyay (2014), Weidner (2013)). Few studies has looked into the IMF of isolated galaxies at $\mathrm{z} \sim 2$ (Quider (2009), Steidel (2004), Pettini (2000)). There are no studies to date which investigates the IMF of galaxies at $\mathrm{z} \sim 2$ using a significant population of galaxies spanning a multitude of physical parameters. This was largely driven by observational constraints due to the absence of sensitive multiplexed Near Infra-red (NIR) spectrographs.

With the introduction of the next generation of multiplexed NIR spectrographs we now have the capability to start exploring large samples of galaxies at $\mathrm{z} \sim 2$ to study their stellar population parameters. ZFIRE is a spectroscopic survey carried out using the MOSFIRE spectrograph in Keck-I aimed at obtaining high quality spectra of rich 
galaxy environments at $\mathrm{z} \sim 2$ (Nanayakkara et al. (in prep)). Using data from the ZFIRE survey, we embark on a journey to study the IMF of a galaxy population at $\mathrm{z} \sim 2$.

\section{The Method}

To place better constraints on the IMF at higher redshifts, galaxies should be observed in situ to understand the underlying physical processors than a relic IMF. To this end we use $\mathrm{H} \alpha \mathrm{EW}$ of star-forming galaxies and their optical colours to constrain the IMF. This method was first developed by Kennicutt (1983). More recently Hoversten (2008) and Gunawardhana (2011) used respectively SDSS and GAMA data to study the IMF of galaxies at $\mathrm{z} \sim 0.1$. Results from SDSS suggested a possibility for a non-universal IMF for the low-luminosity galaxies while results from GAMA showed the possibility for the IMF to vary as a function of star formation rate.

The Physics.

This method relies on basic astrophysics assumptions. The total flux of a galaxy at rest frame $6564.6 \AA$ in vaccum can be considered as its continuum level plus its $\mathrm{H} \alpha$ emission line minus $\mathrm{H} \alpha$ absorption. Reddy (2015) find that $\mathrm{H} \alpha$ absorption for galaxies at $\mathrm{z} \sim 2$ is $\leqslant 3 \%$ of its flux level. Therefore we could safely ignore any effects from absorption.

The remaining continuum and the $\mathrm{H} \alpha$ flux have different physical origins. In the absence of active galactic nuclei, $\mathrm{H} \alpha$ flux is dominated by the ionizing photons emitted in the UV by massive $\mathrm{O}$ and B stars. They are young stars found near ionized Hydrogen regions. If these clouds are optically think (considering a case B recombination), any emitted Lyman continuum photons are immediately reabsorbed. After several scattering events Lyman continuum photons will loose energy and get converted to lower energy photons such as $\mathrm{H} \alpha$. These photons are of low optical depth and therefore can escape the cloud. These transition probabilities are weakly dependent on the temperature and electron density and therefore, it is possible to convert the $\mathrm{H} \alpha$ flux to the number of $\mathrm{O}$ and $\mathrm{B}$ stars in the integrated stellar population. $\mathrm{H} \alpha$ flux should be considered as a lower limit for the amount of $\mathrm{O}$ and $\mathrm{B}$ stars present. This is due to Lyman leakage, which is the possibility of ionizing photons escaping the cloud before being converted to lower series photons. The continuum flux of a galaxy at $6564.6 \AA$ is dominated by red giant stars with masses between $0.7-3 \mathrm{M}_{\odot}$ and the $\mathrm{H} \alpha$ flux comes from stars with masses $>10 \mathrm{M}_{\odot}$. This is shown as a cartoon in Figure 1.

EW can be considered as the ratio of the strength of an emission line to the strength of the continuum at the same wavelength. Therefore the $\mathrm{H} \alpha \mathrm{EW}$ is the ratio of massive $\mathrm{O}$ and $\mathrm{B}$ stars to $\sim 1 \mathrm{M}_{\odot}$ stars which can be used to probe the IMF slope of masses $>1 \mathrm{M}_{\odot}$.

\section{Results}

We define an exponentially varying star-formation history $(\mathrm{SFH})$ as $\mathrm{SFR}(\mathrm{t})=\exp (-$ $\mathrm{t} / \tau) / \tau$, where $t$ is the time $\tau$ is the time constant. In such a SFH, the $\mathrm{H} \alpha \mathrm{EW}$ vs optical colour parameter space can be used to investigate the change of IMF. This is due to the fact that the changes of $\tau$ are orthogonal to the IMF change vector.

After stringent quality cuts, we select a sample of 77 galaxies from the ZFIRE survey at $\mathrm{z} \sim 2$ to calculate the $\mathrm{H} \alpha \mathrm{EW}$ and its $(\mathrm{g}-\mathrm{r})_{0.1}$ colours at $\mathrm{z}=0.1$. We detect the continuum level for 34 galaxies and the remaing 43 galaxies are used as lower limits for the $\mathrm{H} \alpha \mathrm{EW}$. We use the Calzetti (2000) attenuation law to correct the continuum levels and the optical $(\mathrm{g}-\mathrm{r})_{0.1}$ colours. We use the Cardelli (1989) attenuation law to dust correct the nebular emission lines. Attenuation values calculated by FAST are used with the Calzetti (1994) factor to apply a higher amount of extinction to nebular emission line 


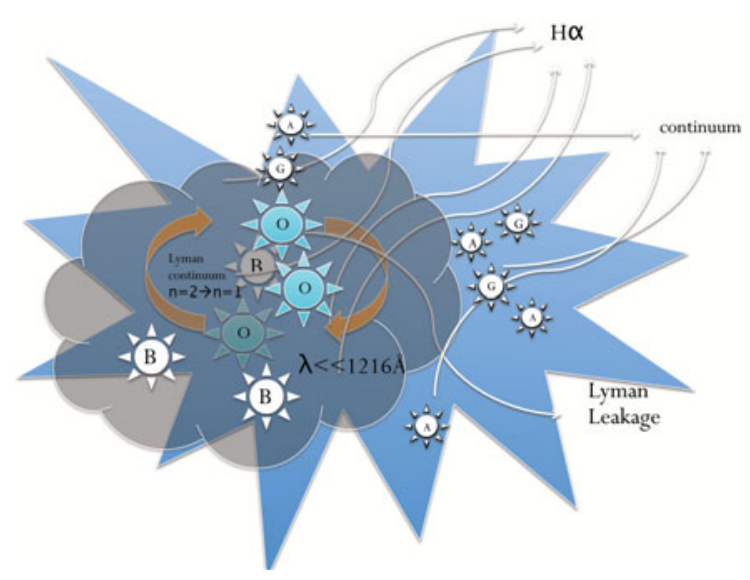

Figure 1. The tale of a molecular cloud. The $\mathrm{H} \alpha$ flux and the continuum level at $6564.6 \AA$ have different physical origins. $\mathrm{H} \alpha$ flux can be used as a direct tracer for the young and massive $\mathrm{O}$ and $\mathrm{B}$ stars in ionized molecular clouds. The continuum contribution at $6564.6 \AA$ is largely driven by the older and smaller A and G stars.

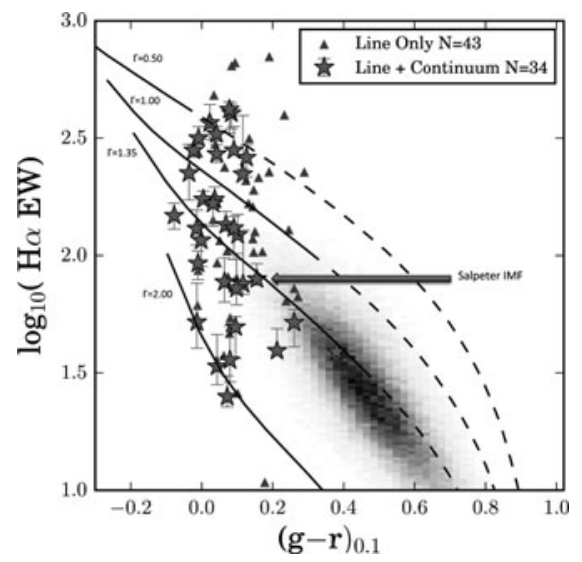

Figure 2. The $\mathrm{H} \alpha \mathrm{EW}$ vs $(\mathrm{g}-\mathrm{r})_{0.1}$ colour distribution of the dust corrected ZFIRE IMF sample. We show the 77 galaxies selected from the COSMOS field here. Galaxies with continuum detections are shown as stars while the triangles represent galaxies with only $\mathrm{H} \alpha$ emission detections. The errors for the continuum detected galaxies are from bootstrap re-sampling. Galaxies from the HG08 sample from SDSS are shown as a 2D histogram. The solid and dashed lines are SSP models computed from PEGASE. All 4 models are computed with the same parameters but with different IMF functions. From top to bottom they have $\Gamma$ values of respectively $0.5,1.0,1.35$ (Similar to Salpeter (1955) value) and 2.0. The solid lines show the evolution of the models up to 3.1 Gyrs $(\mathrm{z} \sim 2)$. The evolution of the models beyond this time period and up to 13 Gyrs $(\mathrm{z} \sim 0)$ is shown as dashed lines.

regions compared to the continuum levels. We use PEGASE (Fioc (1997)) to generate synthetic stellar populations. We define the IMF in the power law form (Bastian (2010) Equation 1) and vary the $\Gamma$ parameter to change the amount of mass within a given logarithmic mass interval.

Figure 2 shows the ZFIRE data along with the SDSS results from Hoversten (2008). The tracks are from PEGASE with a $\tau=1000$ Myr exponentially declining SFH with varying $\Gamma$ values.

Key issues and future work. It is not possible to draw any conclusions about the IMF of our sample yet due to several important factors. The main uncertainty is driven by 
the effect of star-bursts. Star-bursts will increase the $\log \mathrm{H} \alpha \mathrm{EW}$ up to $\sim 0.5$ dex. This change is driven within a short period of time and a thorough understanding of the behaviour is needed to account for this issue. We stack our data and also perform Monte Carlo simulations using PEGASE in order to quantify this effect. The extent up to which the extinction of the continuum vary compared to the nebular emission line is another uncertainty. The ability of SSP models to accurately describe galaxy stellar populations at $\mathrm{z} \sim 2$ is also crucial to interpret the results. These will be addressed in our future paper Nanayakkara et al. (in prep).

Using MOSFIRE we are now able to attempt to infer the IMF of galaxy populations at $\mathrm{z} \sim 2$. With the new generation of NIR instruments, we can now attempt to study these parameters we took for granted to better understand the mysteries of the more distant universe. Using different IMF techniques may lead to inconsistent results between the same galaxy samples (Smith (2014)). Therefore, we finally emphasise that extreme caution is crucial when interpreting IMF of galaxies derived via integrated light techniques.

\section{References}

Baldry, I. K. \& Glazebrook, K. 2003, ApJ, 593, 258

Bastian, N., Covey, K. R., \& Meyer, M. R. 2010, AछAR, 48, 339

Calzetti, D., Armus, L., Bohlin, R. C., et al. 2000, ApJ, 533, 682

Calzetti, D., Kinney, A. L., \& Storchi-Bergmann, T. 1994, ApJ, 429, 582

Cardelli, J. A., Clayton, G. C., \& Mathis, J. S. 1989, ApJ, 345, 245

Cappellari, M., McDermid, R. M., Alatalo, K., et al. 2012, Nature, 484, 485

Chabrier, G. 2003, Publications of the Astronomical Society of the Pacific, 115, pp. 763

Chattopadhyay, T., De, T., Warlu, B., \& Chattopadhyay, A. K. 2015, ApJ, 808, 24

Conroy, C., Dutton, A. A., Graves, G. J., Mendel, J. T., \& van Dokkum, P. G. 2013, Ap. Lett., 776, L26

Ferreras, I., La Barbera, F., de la Rosa, I. G., et al. 2013, MNRAS, 429, L15

Ferreras, I., Weidner, C., Vazdekis, A., \& La Barbera, F. 2015, MNRAS, 448, L82

Fioc, M. \& Rocca-Volmerange, B. 1997, A\& A, 326, 950

Gunawardhana, M. L. P., Hopkins, A. M., Sharp, R. G., et al. 2011, MNRAS, 415, 1647

Hoversten, E. A. \& Glazebrook, K. 2008, ApJ, 675, 163

Kennicutt, Jr., R. C. 1983, ApJ, 272, 54

Kroupa, P. 2001, MNRAS, 322, 231

Meurer, G. R. 2011, in Astronomical Society of the Pacific Conference Series, Vol. 440, UP2010: Have Observations Revealed a Variable Upper End of the Initial Mass Function?, ed. M. Treyer, T. Wyder, J. Neill, M. Seibert, \& J. Lee, 189

Miller, G. E. \& Scalo, J. M. 1979, ApJS, 41, 513

Pettini, M., Steidel, C. C., Adelberger, K. L., Dickinson, M., \& Giavalisco, M. 2000, ApJ, 528, 96

Quider, A. M., Pettini, M., Shapley, A. E., \& Steidel, C. C. 2009, MNRAS, 398, 1263

Reddy, N. A., Kriek, M., Shapley, A. E., et al. 2015, ApJ, 806, 259

Salpeter, E. E., 1955, Apj, Vol. 121, 161

Scalo, J. M. 1986, in IAU Symposium, Vol. 116, Luminous Stars and Associations in Galaxies, ed. C. W. H. De Loore, A. J. Willis, \& P. Laskarides, 451-466

Smith, R. J. 2014, MNRAS, 443, L69

Steidel, C. C., Shapley, A. E., Pettini, M., et al. 2004, ApJ, 604, 534

van Dokkum, P. G. \& Conroy, C. 2010, Nature, 468, 940

Weidner, C., Ferreras, I., Vazdekis, A., \& La Barbera, F. 2013, MNRAS, 435, 2274 